GLYCOBIOLOGY

\section{Telling twins apart}

J. Biol. Chem., published online 13 November

2012, doi:10.1074/jbc.M112.358416

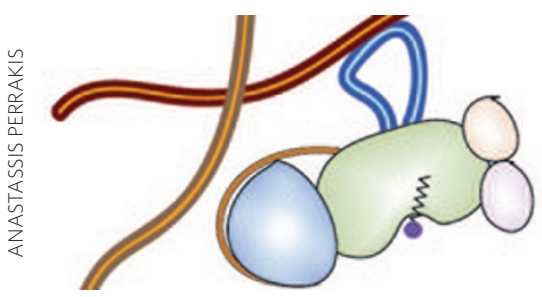

The secreted lysophospholipase autotaxin (ATX) is responsible for synthesizing lysophosphatidic acid (LPA), with numerous cellular outcomes. Most ATX research has focused on the $\beta$ isoform, but three other isoforms are known to be produced, depending on which of the gene's 27 exons are translated. ATX $\alpha$ includes a polybasic 52-residue insert, thought to confer proteolytic instability to the protein. Houben et al. now demonstrate that although the insert is cleaved, most likely at a furin consensus site, this cleavage neither has an impact on protein stability nor affects enzyme activity. The authors also show that ATX $\alpha$ has similar kinetics to ATX $\beta$ and is equally sensitive to an ATX inhibitor, raising the question of why this inserted domain is present. Further inspection of the inserted sequence pointed to the presence of potential heparin-binding motifs; these were validated by the strong interaction of ATX $\alpha$ with unfractionated heparin, with an apparent $K_{\mathrm{d}}$ of $10 \mathrm{nM}$ according to surface plasmon resonance, whereas ATX $\beta$ showed almost no binding. This effect was mirrored by the ability of ATX $\alpha$, but not ATX $\beta$, to bind the surface of glycosaminoglycan-expressing
STRUCTURAL BIOLOGY

\section{E2 loader}

cells in a heparinase-sensitive manner. Surprisingly, heparin also increased ATX $\alpha$ activity by up to two-fold. Though ATX is known to be targeted to the cell membrane by integrin binding, these results point to a second, isoform-specific targeting mechanism that both localizes and activates ATX $\alpha$ to optimize LPA signaling.

\section{$C G$}

CANCER

\section{Feeding forward and back Cancer Cell 22, 585-600 (2012)}

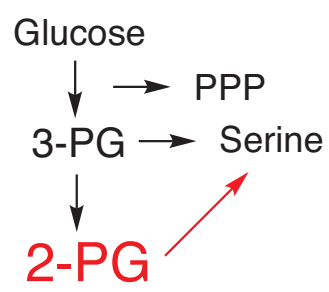

Cancer cells rely on an elevated glycolysis rate for their increased biosynthetic needs. The glycolytic enzyme phosphoglycerate mutase 1 (PGAM1) catalyzes the conversion of 3-phosphoglycerate (3-PG) to 2-PG downstream of most glycolytic reactions that yield biosynthetic precursors. Hitosugi et al. demonstrate that stable knockdown of PGAM1, an enzyme commonly misexpressed in cancer cells, leads to elevated 3-PG and reduced 2-PG in addition to decreasing the rate of glycolysis, flux through the pentosephosphate pathway (PPP) and anabolic biosynthesis. Using biochemical and structural approaches, they showed that 3-PG can directly bind the active site of the PPP enzyme 6-phosphogluconate dehydrogenase (6PGD) and that 3-PG can compete with

Nat. Struct. Mol. Biol. published online 11 November 2012, doi:10.1038/nsmb.2451

Nat. Struct. Mol. Biol. published online 11 November 2012, doi:10.1038/nsmb.2415

Autophagy, a cellular process whereby a double-membrane vesicle engulfs a portion of the cytoplasm and fuses with the lysosome, leading to the degradation of the engulfed contents, is mediated in part by noncanonical ubiquitin-like protein cascades. Atg7, an E1 enzyme, activates the E2 enzymes Atg3 and Atg10. Kaiser et al. and Yamaguchi et al. report crystal structures of the E1-E2 complexes, Atg7-Atg3 and Atg7-Atg10, demonstrating that Atg7 binds both E2 enzymes via the same surface but that the specific amino acids driving the interactions are distinct between the complexes. Both groups also demonstrate in biochemical assays that Atg7 can mediate unexpected conjugation of ubiquitin-like proteins (UBLs) Atg8 and Atg12, transferring them to both E2 enzymes rather than specifying Atg8-Atg3 and Atg12-Atg10 conjugations. Kaiser et al. further confirmed that amino acids essential for Atg7-Atg3 interaction were also necessary to rescue autophagy in $\Delta a t g 7$ or $\Delta a t g 3$ yeast cells. Taken together, these data indicate that E1 enzymes can use the same surface to bind distinct elements from disparate E2 enzymes and that specificity for selective loading of UBLs onto Atg3 and Atg10 observed in vivo might be mediated by elements in addition to Atg7.
6PGD's substrate for binding. Treatment with methyl-2-PG, a compound converted to 2-PG in cells, rescues glycolytic rates and flux through the PPP in cells with stable knockdown of PGAM1, indicating that 2-PG feeds back on upstream steps. Indeed, they found that treatment with methyl-2-PG also decreased 3-PG. Because 3-PG is a precursor for serine biosynthesis, the authors evaluated the effects of 2-PG on 3-phosphoglycerate dehydrogenase (PHGDH), which converts 3-PG to 3-phosphohydroxypyruvate. 2-PG can promote $\mathrm{PHGDH}$ activity in vitro and in cells but only at concentrations higher than those detected in PGAM1 knockdown cells. Taken together, these data indicate that PGAM1 expression controls the amount of 3-PG, which promotes PPP and biosynthesis, and 2-PG, which indirectly controls the amount of 3-PG by regulating $\mathrm{PHGDH}$ activity.

\section{NUCLEAR TRANSPORT}

\section{Bicycle built for two Science 338, 666-671 (2012)}

Ribosome biogenesis occurs in the nucleolus and involves the ordered assembly of rRNA transcripts with ribosomal proteins synthesized in and imported from the cytoplasm. Kressler et al. now show that two ribosomal proteins, Rpl5 and Rpl11, which bind near each other on the $5 S$ rRNA of the $60 S$ ribosomal subunit, are brought into the nucleus together by a newly defined transport system. Using tandem affinity purification, the authors showed that a new protein, symportin 1 (Syo1), purifies together with Rpl5 and Rpl11. Syo1 contains ARM and HEAT repeat domains typically found in nuclear transport proteins and is conserved in eukaryotes. Knockout of yeast syo 1 results in lowered $60 S$ ribosomal subunit abundance, supporting its role in ribosome assembly. Yeast two-hybrid and in vitro reconstitution experiments revealed that Rpl5, Rpl11 and Syo 1 form a heterotrimeric complex. Crystallography and hydrogendeuterium exchange MS defined Syo 1 interactions with Rpl5 and Rpl11, respectively. Though Syo 1 readily transits the nuclear pore complex, the Syo1-Rpl5Rpl11 complex requires Kap104, a nuclear transport receptor, for efficient nuclear import and Ran-GTP for release of the complex from Kap104. Released Syo-Rpl5Rpl11 binds efficiently to 5S rRNA. Taken together, the data support a potentially general model in which coordinated transport of ribosomal proteins can be used to order the assembly of preribosomal complexes. 